Disponible en ligne sur

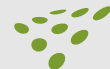

www.sciencedirect.com
Elsevier Masson France

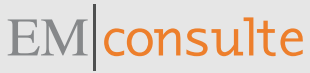

www.em-consulte.com

Revue générale

\title{
La Sensory Modality Assessment and Rehabilitation Technique (SMART) : une échelle comportementale d'évaluation et de revalidation pour des états altérés de conscience
}

\section{The Sensory Modality Assessment and Rehabilitation Technique (SMART): A behavioral assessment scale for disorders of consciousness}

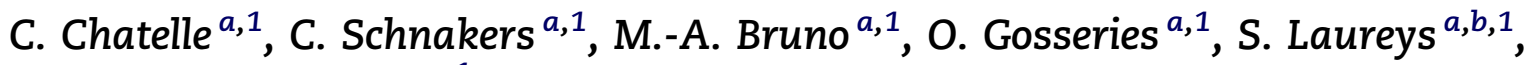
A. Vanhaudenhuyse $e^{a, *, 1}$

${ }^{a}$ Coma Science Group, Cyclotron Research Center, University of Liège, Sart Tilman, B30, 4000 Liège, Belgique

${ }^{\mathrm{b}}$ Département de neurologie, CHU Sart Tilman, Liège, Belgique

I N F O A R T I C L E

Historique de l'article

Reçu le 23 novembre 2009

Reçu sous la forme révisée le

25 décembre 2009

Accepté le 29 janvier 2010

Mots clés :

SMART

Évaluation comportementale

Traitement

État végétatif

État de conscience minimale

Keywords:

SMART

Behavioral assessment

Treatment

Vegetative state

Minimally conscious state

\section{RÉ S U M É}

Introduction. - Le nombre persistant d'erreurs diagnostiques d'état végétatif (40 \%) souligne l'importance d'utiliser des échelles d'évaluation sensibles pour détecter des signes de conscience chez ces patients non communicants.

État des connaissances. - La Sensory Modality Assessment and Rehabilitation Technique (SMART), échelle comportementale utilisant des stimulations sensorielles multimodales, semble être adaptée pour ce type d'évaluation. Plusieurs études ont montré sa sensibilité dans la discrimination de la conscience par rapport à d'autres échelles.

Perspectives. - Cet article présente une description de la procédure SMART, ainsi qu'une revue des études sur sa validité, sa fiabilité et sa robustesse. Une comparaison des réponses évaluées dans la SMART avec celles mesurées dans d'autres échelles standardisées validées est également proposée.

Conclusions. - Malgré certaines limites, la SMART semble adéquate pour un suivi à long terme des patients en état de conscience altérée mais son utilité en revalidation nécessite l'investigation de l'effet des stimulations sur l'évolution des patients. Étudier sa validité concourante avec d'autres échelles validées semble aussi nécessaire.

(C) 2010 Elsevier Masson SAS. Tous droits réservés.

A B S T R A C T

Introduction. - Difficulties in detecting bedside signs of consciousness in non-communicative patients still lead to a high rate of misdiagnosis illustrating the need to employ standardized behavioral assessment scales.

\footnotetext{
* Auteur correspondant.

Adresse e-mail : steven.laureys@ulg.ac.be (S. Laureys), avanhaudenhuyse@doct.ulg.ac.be, avanhaudenhuyse@student.ulg.ac.be

(A. Vanhaudenhuyse).

${ }^{1}$ http://www.comascience.org.

0035-3787/\$ - see front matter (C) 2010 Elsevier Masson SAS. Tous droits réservés.

doi:10.1016/j.neurol.2010.01.011
} 
State of art. - The Sensory Modality Assessment and Rehabilitation Technique (SMART) is a behavioral assessment scale of consciousness that assesses responses to multimodal sensory stimulation in disorders of consciousness. These stimulations can also be considered to have therapeutic value.

Perspectives. - We here review the different components and use of the SMART assessment and discuss its validity, reliability, and robustness in clinical practice. The scale has a high intra- and inter-observer reliability thanks to a detailed procedure description. However, in the absence of objective gold standards in the assessment of consciousness, it is currently difficult to make strong claims about its validity. A comparison between SMART and other standardized and validated coma-scales is proposed.

Conclusion. - In our view, SMART is an interesting tool for monitoring patients with altered states of consciousness subsequent to coma. Currently, we await studies on its concurrent validity as compared to other validated behavioral assessment scales and on the effect of SMART stimulations on patient outcome.

(C) 2010 Elsevier Masson SAS. All rights reserved.

\section{Introduction}

Les progrès en médecine concernant la réanimation et les soins ont engendré une augmentation du nombre de patients survivant à de graves lésions cérébrales. Certains se rétablissent rapidement, tandis que d'autres traversent différents états tels que le coma, l'état végétatif et l'état de conscience minimale (pour une revue : Laureys et al., 2004). L'état végétatif est défini comme la récupération du cycle veille-sommeil en l'absence d'une réponse consciente, seules des réponses réflexes pouvant être observées (The Multi-Society Task Force on PVS, 1994). L'état de conscience minimale se caractérise par des comportements conscients et volontaires, bien que ceuxci soient inconsistants et élémentaires, tels qu'une réponse à une commande simple, une poursuite visuelle et/ou une localisation de stimulations nociceptives ; sans communication fonctionnelle possible (Giacino et al., 2002). L'émergence de l'état de conscience minimale est caractérisée par la capacité du patient à communiquer ou à utiliser des objets de manière fonctionnelle (Giacino et al., 2002). Distinguer un patient en état végétatif d'un patient en état de conscience minimale demeure un véritable défi. En effet, 30 à $40 \%$ des patients sont encore diagnostiqués étant en état végétatif alors qu'ils sont en réalité conscients (Schnakers et al., 2009) et ce, malgré l'introduction de nouveaux critères diagnostiques (Giacino et al., 2002). Ces résultats reflètent le manque de sensibilité des outils utilisés dans l'évaluation comportementale de la conscience. Actuellement, la Coma Recovery ScaleRevised (CRS-R - Giacino et al., 2004) a montré sa sensibilité par rapport aux autres échelles, celle-ci permettant de diminuer significativement l'erreur diagnostique (Schnakers et al., 2006, 2008). Elle semble selon nous être actuellement la référence standard pour l'évaluation comportementale de la conscience. Dans cet article, nous présenterons la Sensory Modality Assessment and Rehabilitation Technique (SMART - GillThwaites, 1997 ; Gill-Thwaites et Munday, 1999), une échelle qui utilise des stimulations variées et multimodales et qui a montré son utilité dans la prise en charge clinique des patients en état de conscience altérée (Gill-Thwaites et Munday, 2004 ; Wilson et Gill-Thwaites, 2000). Nous comparerons cette échelle avec la CRS-R et d'autres échelles couramment utilisées en clinique telles que la Full Outline of Unresponsiveness (FOUR - Wijdicks et al., 2005), la Wessex Head Injury Matrix (WHIM - Shiel et al., 2000), la Glasgow Liège Scale (GLS Born, 1988) et la Western Neuro Sensory Stimulation Profile (WNSSP - Ansell et Keenan, 1989).

\section{SMART : une échelle d'évaluation et de traitement}

La SMART a été créée par l'équipe de thérapie occupationnelle du Royal Hospital for Neuro-disability en 1988 et se base sur les paramètres de la Glasgow Coma Scale (Teasdale et Jennett, 1974). La procédure SMART est composée de deux types d'évaluation : l'une est informelle et l'autre formelle (Tableau 1). L'évaluation informelle comporte deux questionnaires remplis à la fois par la famille, les proches et par l'équipe soignante. Le premier questionnaire traite des habitudes de vie (goûts alimentaires, choix musicaux, etc.) du patient durant la période précédant son accident. Ces informations seront utiles pour l'évaluation et orienteront le traitement proposé. Il y est demandé d'indiquer ce que le patient aimait ou n'aimait pas avant son accident (musique, nourriture, etc.) afin d'évaluer l'intérêt des stimulations intégrées dans le protocole SMART. Le second questionnaire, proposé à toutes personnes étant en contact avec le patient, résume l'ensemble des comportements et réactions motrices observés chez le patient au cours de l'hospitalisation. Une feuille détaillée permet de définir la date, l'heure, la stimulation ayant provoqué la réponse observée, sa fréquence et sa durée.

Les différents comportements sont ainsi notés systématiquement et permettent d'optimaliser la seconde partie de l'évaluation (l'administration formelle de la SMART). L'évaluation formelle (de la SMART) est réalisée par un clinicien entraîné et consiste à évaluer les réponses comportementales à différentes stimulations. Après avoir observé les comportements spontanés du patient durant dix minutes (ligne de base), les réactions observées suite aux différentes stimulations administrées seront notées et rapportées par le clinicien. En plus d'être une échelle d'évaluation, l'intérêt principal de la SMART est qu'elle peut être administrée en tant qu'outil 
Tableau 1 - Protocole d'évaluation et de traitement SMART (adapté de Gill-Thwaites et Munday, 1999). SMART Assessment and Treatment Protocol (adapted from Gill-Thwaites et Munday, 1999).

\begin{tabular}{|c|c|c|c|}
\hline Étapes programme SMART & Durée et fréquence & Procédure & Responsabilités \\
\hline 1 : avant évaluation & $\begin{array}{l}\text { À l'admission du patient ou } \\
\text { précédant l'évaluation }\end{array}$ & $\begin{array}{l}\text { Questionnaire sur les } \\
\text { habitudes du patient avant l'accident } \\
\text { Observation des comportements }\end{array}$ & $\begin{array}{l}\text { Famille et personnel } \\
\text { soignant }\end{array}$ \\
\hline 2 : évaluation SMART & $\begin{array}{l}\text { Durant } 3 \text { semaines } \\
10 \text { sessions d'évaluation réparties } \\
\text { en " matin " et " après-midi » }\end{array}$ & $\begin{array}{l}\text { Observation des } \\
\text { comportements spontanés } \times 10 \\
\text { SMART } \times 10\end{array}$ & Examinateur \\
\hline 3 : bilan & Après 10 sessions d'évaluation & $\begin{array}{l}\text { Mise en place du traitement } \\
\text { sur base des observations }\end{array}$ & Examinateur \\
\hline $\begin{array}{l}\text { 4: programme de } \\
\text { traitement SMART }\end{array}$ & $\begin{array}{l}8 \text { semaines (journalier) ou jusqu'à } \\
\text { obtention du niveau } 5 \text { dans une } \\
\text { des modalités (à } 5 \text { moments } \\
\text { consécutifs), excepté la vigilance }\end{array}$ & $\begin{array}{l}\text { Traitement SMART et } \\
\text { observation des comportements }\end{array}$ & $\begin{array}{l}\text { Examinateur } \\
\text { Famille et personnel } \\
\text { soignant }\end{array}$ \\
\hline 5 : révision du traitement & $\begin{array}{l}\text { À l'obtention du niveau } 5 \text { dans } \\
\text { une modalité ou tous les } 2 \text { mois } \\
\text { durant } 6 \text { mois, puis tous les } 6 \text { mois }\end{array}$ & $\begin{array}{l}\text { Processus d'évaluation SMART } \\
\text { et révision du traitement selon } \\
\text { les observations }\end{array}$ & $\begin{array}{l}\text { Examinateur } \\
\text { Famille et personnel } \\
\text { soignant }\end{array}$ \\
\hline $\begin{array}{l}6 \text { : développement d'un } \\
\text { système de communication }\end{array}$ & $\begin{array}{l}\text { Durée et moment définis par } \\
\text { l'équipe selon l'évolution observée }\end{array}$ & $\begin{array}{l}\text { Mise en place d'un système de } \\
\text { communication fonctionnelle }\end{array}$ & $\begin{array}{l}\text { Examinateur } \\
\text { Famille et personnel } \\
\text { soignant }\end{array}$ \\
\hline
\end{tabular}

thérapeutique de stimulation sensorielle. Cependant, aucune étude n'a pu mettre en évidence l'effet à long terme de ces stimulations sur l'évolution de patients en état de conscience altérée.

L'évaluation formelle permet de situer l'état du patient et d'obtenir des informations sur ses capacités motrices, communicationnelles et sensorielles. Les observations faites durant ces évaluations influenceront la phase de traitement. Le patient est évalué sur dix sessions journalières pendant trois semaines ; cinq évaluations se font le matin et cinq l'après-midi, cela afin de déterminer la période durant laquelle le patient est le plus répondant. Après les dix évaluations, le traitement sera mis en place sur base des observations des cliniciens.

L'examinateur décidera de stimuler une modalité en particulier si le patient se montre particulièrement réactif à ce type de stimulation durant la période d'évaluation. Par ailleurs, les stimulations peuvent intégrer du matériel plus personnel, comme par exemple des photographies de proches, des odeurs particulières, etc. Cette phase de traitement a lieu au minimum une fois par jour pendant au moins deux mois. Elle dure environ 30 minutes et toutes les réponses sont notées.

Avant de commencer l'administration de la SMART, l'examinateur se présente au patient et donne des informations spatiotemporelles. Celles-ci seront répétées à la fin de la session. Dix minutes d'observation sans stimulation sont nécessaires avant de débuter l'évaluation (ligne de base). La SMART évalue huit modalités : cinq modalités sensorielles (visuelle, tactile, auditive, olfactive et gustative), la motricité, la communication fonctionnelle ainsi que la vigilance. Pour chaque modalité, le protocole décrit explicitement les instructions à donner aux patients, le matériel utilisé, la position de l'examinateur, les observations à faire, ainsi que quelques conseils à prendre en compte durant l'évaluation (exemple : en cas de cécité, paralysie).
Les conditions engendrant un arrêt ou la non-administration d'une partie de l'échelle sont précisées (patient atteint de cécité, de surdité, paralysie, allergies, trachéotomie, etc.). Au cours de l'évaluation, si le patient répond aux indications écrites et non aux indications orales, l'examinateur devra utiliser la modalité visuelle. Chaque stimulation est précédée de dix secondes et suivie de 20 secondes d'observation et ce, afin de distinguer les comportements réflexes des comportements volontaires. Un total de dix évaluations est nécessaire avant d'effectuer un avis quant au diagnostic. Les auteurs suggèrent de varier l'ordre de présentation des différentes modalités. Un code précis est utilisé afin de coter les réponses observées ; pas de réponse se code par [-], réponse inexacte par [In], réponse exacte par [x], réponse retardée par [NR] et ambiguë par [?]. Le côté où apparaît la réponse doit aussi être noté (gauche ou droit). Le Tableau 2 reprend les différents comportements observés selon les modalités.

Étant une échelle graduelle, le niveau de cotation augmente avec la complexité des réponses observées. Cinq niveaux sont décrits :

- niveau 1 : pas de réponse : aucune réponse ne peut être observée ;

- niveau 2 : réponses réflexes : réponses stéréotypées et réflexes (flexion, extension ou sursaut) ;

- niveau 3 : retrait : mouvements de retrait ;

- niveau 4 : localisation : réponses orientées ;

- niveau 5 : réponses différenciées : réponses adéquates à une commande visuelle ou verbale ; utilisation fonctionnelle d'objet.

La SMART ne permet pas d'émettre un diagnostic uniquement sur la base des niveaux de réponses du patient. En effet, les réponses de niveau 1, 2 ou 3 peuvent être observées chez les patients en coma ou en état végétatif. Les réponses de niveau 4 peuvent être présentes chez un patient en état végétatif et en 
Tableau 2 - Résumé des différents comportements observés pour chaque modalité de la SMART. Summary of the observed behaviors in SMART modalities.

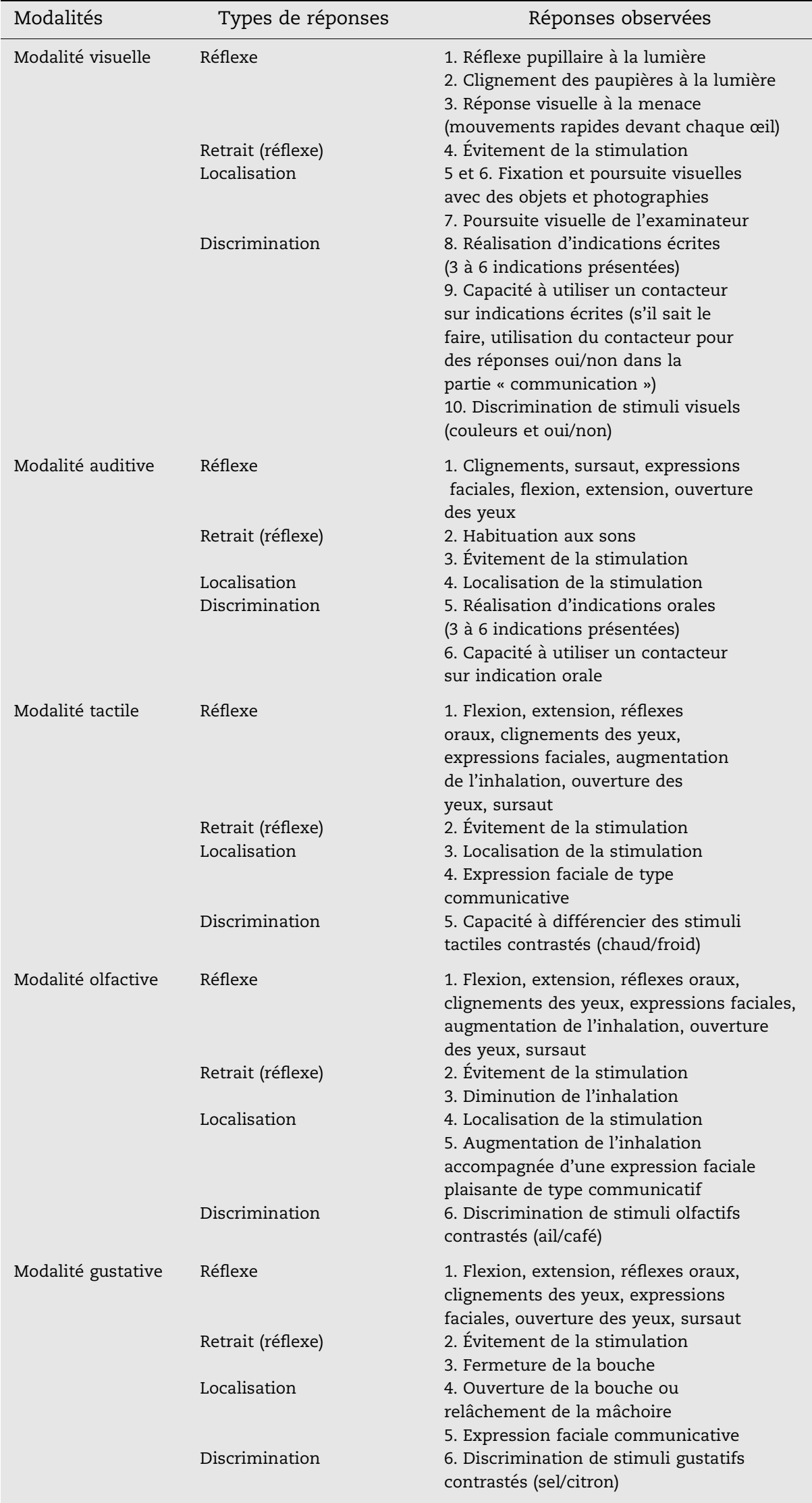

Niveau 1 : absence de réponse Niveau 2 : présence de comportements inclus dans (1) Niveau 3 : (2) ou (3) présents Niveau 4 : présence de (4) Niveau 5 : réalisation de (5) ou (6)

Niveau 1 : absence de réponse Niveau 2 : présence des comportements inclus dans (1) Niveau 3 : présence de (2) Niveau 4 : présence de (3) Niveau 5 : (5) réalisé

Niveau 1 : absence de réponse Niveau 2 : présence des comportements inclus dans (1) Niveau 3 : présence de (2) ou (3) Niveau 4 : présence de (4) ou (5) Niveau 5 : (6) réalisé

Niveau $1:$ absence de réponse Niveau 2 : présence des comportements inclus dans (1) Niveau 3 : présence de (2) et (3) Niveau 4 : présence de (4) ou (5) Niveau 5 : réalisation de (6) 


\begin{tabular}{|c|c|c|c|}
\hline Modalités & Types de réponses & Réponses observées & Cotation \\
\hline Modalité motrice & $\begin{array}{l}\text { Réflexe } \\
\text { Mouvement non } \\
\text { contextualisé/retrait } \\
\text { Mouvement } \\
\text { contextualisé/localisation } \\
\text { ( }<5 \text { observations) }\end{array}$ & $\begin{array}{l}\text { 1. Flexion, extension, grasping, } \\
\text { clignements des yeux } \\
\text { 2. Mouvements présents en } \\
\text { l'absence de stimulation } \\
\text { 3. Utilisation non fonctionnelle d'objets } \\
\text { 4. Évitement de la stimulation } \\
\text { 5. Réponses aux commandes } \\
\text { visuelles/orales } \\
\text { 6. Utilisation fonctionnelle } \\
\text { inconsistante d'objets } \\
\text { 7. Mouvement de résistance ou de } \\
\text { rejet face à la stimulation } \\
\text { 8. Localisation de la stimulation } \\
\text { 9. Réponses consistantes aux } \\
\text { commandes visuelles/orales } \\
\text { 10. Utilisation fonctionnelle et } \\
\text { consistante d'objets }\end{array}$ & $\begin{array}{l}\text { Niveau } 1: \text { absence de réponse } \\
\text { Niveau } 2: \text { présence des } \\
\text { comportements inclus dans (1) } \\
\text { Niveau } 3: \text { présence de (2), (3) ou (4) } \\
\text { Niveau } 4 \text { : présence de (5), (6), (7) ou (8) } \\
\text { Niveau } 5 \text { atteint lorsque les items } \\
\text { (9) ou (10) sont réalisés }\end{array}$ \\
\hline Communication & $\begin{array}{l}\text { Communication } \\
\text { inconsistante } \\
\text { ( }<5 \text { observations) } \\
\text { Communication } \\
\text { consistante } \\
\text { ( }>5 \text { observations) }\end{array}$ & $\begin{array}{l}\text { 1. Vocalisations, expressions } \\
\text { faciales, rires non spécifiques à } \\
\text { la stimulation } \\
\text { 2. Vocalisations, expressions } \\
\text { faciales, rires, gestes spécifiques à } \\
\text { la stimulation } \\
\text { 3. Gestes, expressions faciales, } \\
\text { verbalisations inconsistants avec } \\
\text { les stimulations } \\
\text { 4. Réponses oui/non inconsistantes } \\
\text { dans les différentes modalités } \\
\text { 5. Gestes, expressions faciales, } \\
\text { verbalisations consistants avec } \\
\text { les stimulations } \\
\text { 6. Réponses oui/non consistantes } \\
\text { dans les différentes modalités } \\
\text { 7. Expression par écrit ou via un buzzer }\end{array}$ & $\begin{array}{l}\text { Niveau } 1 \text { : absence de réponse } \\
\text { Niveau } 2: \text { présence des } \\
\text { comportements inclus dans (1) } \\
\text { Niveau } 3: \text { présence de (2) } \\
\text { Niveau } 4: \text { présence de (3) ou (4) } \\
\text { Niveau } 5 \text { : réalisation de (5), (6) ou (7) }\end{array}$ \\
\hline Modalité vigilance & $\begin{array}{l}\text { Aucun éveil } \\
\text { Éveil minimal } \\
\text { Éveil moyen } \\
\text { Éveil normal }\end{array}$ & $\begin{array}{l}\text { 1. Yeux clos durant toute l'évaluation } \\
\text { 2. Plus de } 5 \text { stimulations nécessaires } \\
\text { pour maintenir l'éveil } \\
\text { 3. } 2-4 \text { stimulations nécessaires } \\
\text { pour maintenir l'éveil } \\
\text { 4. } 1 \text { stimulation nécessaire pour } \\
\text { maintenir l'éveil } \\
\text { 5. Éveil constant }\end{array}$ & $\begin{array}{l}\text { Niveau } 1 \text { : présence de (1) } \\
\text { Niveau } 2: \text { nécessité de (2) } \\
\text { Niveau } 3: \text { nécessité de (3) } \\
\text { Niveau } 4: \text { nécessité de (4) } \\
\text { Niveau } 5 \text { : présence de (5) }\end{array}$ \\
\hline
\end{tabular}

état de conscience minimale, alors qu'un patient en état de conscience minimale ou émergeant de cet état présente des comportements de niveau 5 (Tableau 3).

Le score total ne se compose pas de l'ensemble des scores dans les différentes modalités ; chaque modalité est considérée individuellement comme un score unique. Lorsqu'une réponse du niveau 5 (voir paragraphe suivant) apparaît comme constante au cours de cinq évaluations consécutives dans une modalité (excepté la vigilance), on peut conclure à une récupération de la conscience. Le traitement peut être modifié ou arrêté si les réponses aux stimulations sont constantes et significatives. À l'inverse, l'absence d'une réponse constante de niveau 5 mènera à une prolongation de deux mois avec révision tous les deux mois durant six mois, ensuite le programme sera revu tous les six mois. Selon l'évolution du patient, les cliniciens vont ensuite mettre en place un système de communication, cela en collaboration avec les proches et le personnel soignant.

\section{Sensibilité et fiabilité de la SMART}

Lors d'une étude réalisée auprès de 30 patients en état végétatif comparant la WNSSP, la SMART ainsi que la Rancho Levels of Cognitive Functioning (Rancho - Gill-Thwaites, 1997 ; Malkmus et al., 1980), les auteurs ont mis en évidence que la SMART et la Rancho différaient significativement quant au diagnostic. Les résultats démontraient que la SMART détectait un fonctionnement cognitif plus élevé chez les patients. De plus, sur six patients détectés avec la SMART comme émergeant de l'état végétatif au cours de l'étude, seulement deux l'ont été avec la WNSSP. Ces résultats peuvent s'expliquer par le fait que, contrairement à la WNSSP, la SMART comporte différentes modalités, chacune examinée indépendamment des autres. Chaque score a donc une valeur indépendante et l'évaluation globale ne consiste pas en une addition des scores mais en l'interprétation de ceux-ci pour chaque modalité. 
Tableau 3 - Niveaux définis par la SMART, diagnostic et traits cliniques associés. SMART levels, diagnosis and clinical features.

Niveaux

$\begin{array}{ll}1 & \text { Coma } \\ 1 & \text { EV } \\ 2-3 & \text { Coma } \\ 2-3 & \text { EV } \\ 4 & \text { EV/ECM } \\ 5 & \text { ECM/émergence de l'ECM }\end{array}$

Traits cliniques

Yeux fermés. Pas de réponse

Yeux ouverts. Pas de réponse

Yeux fermés. Mouvements réflexes à la stimulation nociceptive. Pas d'autre réponse

Yeux ouverts. Réponses réflexes et de retrait

Comportement montrant un ECM. Nécessite l'établissement de la qualité et consistance des réponses pour définir si EV ou ECM Réponse consistante indiquant un niveau de conscience. Sortie de l'EV. Nécessite d'autres évaluations pour établir les possibilités futures

EV : état végétatif ; ECM : état de conscience minimale.

Par ailleurs, Wilson et Gill-Thwaites (2000) ont démontré l'intérêt des scores totaux de la SMART dans le pronostic des patients en état végétatif. Une différence significative a été observée dans les scores obtenus chez les patients végétatifs selon qu'ils avaient récupéré au cours de l'étude ou non. Ce constat n'était pas généralisable à la WNSSP. Les auteurs expliquaient cela par le fait que la WNSSP considère toutes les réponses moins complexes que la localisation (niveau 4 dans la SMART) comme une absence de réponse. Ajoutons que l'importance du score pour chaque modalité est également répartie dans la SMART, alors que dans la WNSSP, la modalité visuelle a plus d'importance dans le score total. Andrews et al. (1996) ont démontré que $65 \%$ des patients ayant été mal diagnostiqués étaient aveugles ou fortement atteints visuellement.

Enfin, une bonne fiabilité intra- et interjuges a été rapportée pour la SMART, la précision de ses items limitant les variations trop importantes dans la cotation (Gill-Thwaites et Munday, 2004). Dans cette étude, deux examinateurs ont évalué 60 patients diagnostiqués en état végétatif à l'admission, et cela dans la première semaine et tous les deux mois à partir de leur hospitalisation. La corrélation intrajuge obtenue était de 0,97 , et la corrélation interjuge, de 0,96. Ces résultats démontrent une faible variabilité dans les scores obtenus entre évaluateurs et sur plusieurs évaluations effectuées par un même évaluateur. La validité est en revanche difficile à apprécier, étant donné qu'il n'y a pas de consensus concernant une échelle de référence commune. Cependant, étant donné que la CRS-R a montré une plus grande sensibilité par rapport au diagnostic, il semblerait utile de comparer la SMART à cette dernière.

\section{Comparaison entre la SMART et d'autres échelles standardisées et validées}

Tout comme la CRS-R, la FOUR, la WHIM, la GLS et la WNSSP, la SMART évalue la poursuite visuelle qui est considérée comme un des premiers signes de conscience réapparaissant chez les patients sévèrement cérébrolésés (Giacino et al., 2002) (Tableau 4). La fixation est également reprise dans chacune de ces échelles sauf dans la FOUR et la WNSSP. Cependant, la SMART permet à l'examinateur d'utiliser diverses stimulations pour évaluer la poursuite visuelle des patients, alors que la CRS-R insiste sur l'utilisation d'un miroir, outil ayant été démontré comme significativement plus sensible pour détecter ce comportement (Vanhaudenhuyse et al., 2008). Dans la modalité auditive, la SMART est la seule échelle à évaluer les comportements d'habituation et de retrait par rapport à une source sonore. Néanmoins, aucune étude jusqu'à ce jour n'a démontré l'utilité de ces items pour le diagnostic des patients en état de conscience altérée. Notons que la SMART et la WNSSP évaluent toutes deux les réponses du patient à une stimulation tactile non douloureuse, mais elles ne s'intéressent pas aux réponses à la stimulation tactile douloureuse. Ce type de stimulus est utilisé dans la CRS-R, la FOUR et la GLS. Aucune donnée ne permet de valider l'utilisation d'une stimulation douloureuse dans l'évaluation de la conscience. Nous pouvons également remarquer que la SMART et la WNSSP évaluent les réponses à des stimulations olfactives, la SMART utilise aussi les stimulations gustatives alors que les autres n'en tiennent pas compte. L'intérêt de ces modalités dans l'évaluation de la conscience chez des patients sévèrement cérébrolésés n'a cependant pas encore été démontré. Enfin, la CRS-R est la seule échelle à évaluer tous les réflexes du tronc cérébral (réflexes fronto-orbiculaire, pupillaire, cornéen, oculocéphaliques verticaux/horizontaux, oculocardiaque et nauséeux ; les mouvements spontanés des yeux et les réponses stéréotypées). La SMART, quant à elle, ne s'intéresse qu'aux réflexes pupillaires et aux réponses stéréotypées. Cette évaluation a toute son importance chez les patients en état de conscience altérée car elle permet de détecter s'il y a des lésions structurelles au niveau du circuit de l'éveil, les fonctions du tronc ainsi que les circuits moteurs étant adjacents à ce système. Les réponses oculomotrices sont particulièrement informatives quant à la préservation du système d'éveil (Posner et al., 2007).

Un danger de la SMART est qu'une seule réponse est suffisante pour atteindre un niveau, et bien que cela offre une information sur la meilleure réponse du sujet, cette dernière peut aussi être due au hasard (exemple : contraction involontaire de la main du patient apparaissant lorsque l'examinateur lui demande de lui serrer la main). La SMART a en revanche comme atout le nombre important de stimulations et modalités utilisées et la prise en compte des informations provenant des proches. Cela permet l'utilisation de stimulations, personnalisées ou non, comme traitement, ce qui n'est pas envisageable avec les autres échelles, bien qu'il n'y ait pas encore eu à ce jour d'étude sur les effets réels de ces stimulations sur l'évolution de la conscience de chez ces patients. 
Tableau 4 - Tableau comparatif des comportements observés dans la SMART (Gill-Thwaites et Munday, 1999), la Coma Recovery Scale-Revised (CRS-R, Giacino et al., 2004), la Full Outline of Unresponsiveness (FOUR, Wijdicks et al., 2005), la Wessex Head Injury Matrix (WHIM, Shiel et al., 2000), la Glasgow Liège Scale (GLS, Born, 1988) et la Western Neuro Sensory Stimulation Profile (WNSSP, Ansell et Keenan, 1989).

Comparison table between SMART, CRS-R, FOUR, WHIM, GLS and WNSSP for behavioral responses assessed.

\begin{tabular}{|c|c|c|c|c|c|c|c|}
\hline & Réponses comportementales & SMART & CRS-R & FOUR & WHIM & GLS & WNSSP \\
\hline Vigilance & & $\mathrm{X}$ & $\mathrm{X}$ & $\mathrm{X}$ & $\mathrm{X}$ & $\mathrm{x}$ & $\mathrm{X}$ \\
\hline \multirow[t]{6}{*}{ Visuel } & Ouverture des yeux/éveil & $\mathrm{X}$ & $\mathrm{X}$ & $\mathrm{X}$ & $\mathrm{X}$ & $\mathrm{x}$ & $\mathrm{X}$ \\
\hline & Réflexe de clignement à la menace & $\mathrm{X}$ & $\mathrm{X}$ & & & & \\
\hline & Fixation & $\mathrm{X}$ & $\mathrm{X}$ & & $\mathrm{X}$ & & \\
\hline & Poursuite visuelle & $\mathrm{X}$ & $\mathrm{X}$ & $\mathrm{X}$ & $\mathrm{X}$ & & $\mathrm{X}$ \\
\hline & Discrimination visuelle & $\mathrm{X}$ & $\mathrm{X}$ & & $\mathrm{X}$ & & \\
\hline & Compréhension de commande visuelle & $\mathrm{X}$ & & & & & $\mathrm{x}$ \\
\hline \multirow[t]{5}{*}{ Auditif } & Réflexe de sursaut au bruit & $\mathrm{X}$ & $\mathrm{X}$ & $\mathrm{X}$ & & $\mathrm{X}$ & $\mathrm{X}$ \\
\hline & Habituation au bruit & $\mathrm{X}$ & & & & & \\
\hline & Mouvement de retrait & $\mathrm{X}$ & & & & & \\
\hline & Localisation de son & $\mathrm{X}$ & $\mathrm{X}$ & & $\mathrm{X}$ & & $\mathrm{X}$ \\
\hline & Compréhension de commande & $\mathrm{x}$ & $\mathrm{X}$ & $\mathrm{X}$ & $\mathrm{X}$ & $\mathrm{X}$ & $\mathrm{x}$ \\
\hline \multirow[t]{6}{*}{ Verbal/Communication } & Réflexes oraux & $\mathrm{x}$ & $\mathrm{X}$ & & $\mathrm{X}$ & & \\
\hline & Vocalisation/mouvements oraux & $\mathrm{X}$ & $\mathrm{X}$ & & $\mathrm{X}$ & $\mathrm{X}$ & $\mathrm{X}$ \\
\hline & Verbalisation intelligible & $\mathrm{X}$ & $\mathrm{X}$ & & $\mathrm{X}$ & $\mathrm{X}$ & $\mathrm{X}$ \\
\hline & Communication non fonctionnelle intentionnelle & $\mathrm{X}$ & $\mathrm{X}$ & & $\mathrm{x}$ & $\mathrm{X}$ & $\mathrm{X}$ \\
\hline & Communication fonctionnelle intentionnelle & $\mathrm{x}$ & $\mathrm{x}$ & & $\mathrm{x}$ & $\mathrm{x}$ & \\
\hline & Orientation & & & & $\mathrm{x}$ & $\mathrm{x}$ & \\
\hline \multirow[t]{4}{*}{ Tactile (non douloureux) } & Réponses stéréotypées & $\mathrm{X}$ & & & & & $\mathrm{x}$ \\
\hline & Flexion & $\mathrm{X}$ & & & & & $\mathrm{X}$ \\
\hline & Localisation & $\mathrm{X}$ & & & & & $\mathrm{X}$ \\
\hline & Discrimination & $\mathrm{x}$ & & & & & \\
\hline \multirow[t]{3}{*}{ Tactile nociceptif } & Réponses stéréotypées & & $\mathrm{x}$ & $\mathrm{x}$ & & $\mathrm{x}$ & \\
\hline & Flexion & & $\mathrm{X}$ & $\mathrm{X}$ & & $\mathrm{x}$ & \\
\hline & Localisation & & $\mathrm{x}$ & $\mathrm{x}$ & & $\mathrm{x}$ & \\
\hline \multirow[t]{4}{*}{ Olfactif } & Réponses réflexes & $\mathrm{X}$ & & & & & $\mathrm{X}$ \\
\hline & Mouvement de retrait & $\mathrm{X}$ & & & & & \\
\hline & Localisation & $\mathrm{X}$ & & & & & \\
\hline & Discrimination & $\mathrm{x}$ & & & & & $\mathrm{x}$ \\
\hline \multirow[t]{4}{*}{ Gustatif } & Réponses réflexes & $\mathrm{x}$ & & & & & \\
\hline & Mouvement de retrait & $\mathrm{X}$ & & & & & \\
\hline & Localisation & $\mathrm{X}$ & & & & & \\
\hline & Discrimination & $\mathrm{X}$ & & & & & \\
\hline Respiration & & & & $\mathrm{x}$ & & & \\
\hline \multirow[t]{9}{*}{ Réflexes du tronc cérébral } & Fronto-orbiculaire & & $\mathrm{x}$ & & & $\mathrm{x}$ & \\
\hline & Pupillaire & $\mathrm{X}$ & $\mathrm{x}$ & $\mathrm{X}$ & & $\mathrm{x}$ & \\
\hline & Cornéen & & $\mathrm{X}$ & $\mathrm{X}$ & & & \\
\hline & Mouvements spontanés des yeux & & $\mathrm{X}$ & & & & \\
\hline & Oculocéphalique vertical & & $\mathrm{X}$ & & & $\mathrm{x}$ & \\
\hline & Oculocéphalique horizontal & & $\mathrm{X}$ & & & $\mathrm{X}$ & \\
\hline & Réponses stéréotypées & $\mathrm{x}$ & $\mathrm{x}$ & $\mathrm{X}$ & & $\mathrm{x}$ & \\
\hline & Oculocardiaque & & & & & $\mathrm{x}$ & \\
\hline & Nauséeux & & $\mathrm{X}$ & $\mathrm{X}$ & & & \\
\hline
\end{tabular}

\section{Comment devenir un examinateur SMART ?}

Une formation SMART est proposée par l'institut de réadaptation neuropalliative à Londres. Elle est payante et obligatoire pour toutes personnes désirant utiliser cette échelle. Un certain nombre de qualités d'observation et de compétences cliniques est requis pour la réussite de cette formation. La participation aux cours ainsi qu'un travail pratique permet- tront aux participants de devenir des examinateurs SMART. Une compréhension de l'anglais est indispensable pour participer à la formation, dans le cas contraire, une alternative doit être envisagée avec les formateurs. La formation dure cinq jours. Une première journée introduit l'évaluation et le traitement chez les patients en état végétatif et en état de conscience minimale. Les quatre autres journées de cours permettront un apprentissage approfondi du protocole de la SMART comme évaluation et comme traitement. 


\section{Conclusion}

Des études ont montré que la SMART était plus sensible que la WNSSP et la Rancho dans l'évaluation des patients récupérant de coma. Cependant, aucune étude n'a actuellement été réalisée comparant la SMART à la CRS-R, échelle qui a pourtant montré sa sensibilité par rapport à d'autres échelles telles que la WHIM et la FOUR dans la détection de signe de conscience. La SMART semble, par ailleurs, utile dans le suivi des patients à long terme et en revalidation. Elle permet d'évaluer les réponses du patient à un nombre élevé de stimulations sensorielles, et cela dans différentes modalités, toutes cotées indépendamment les unes des autres. Ces mêmes stimulations peuvent aussi être utilisées par la suite dans une phase de traitement. Le protocole de la SMART est précis et détaillé quant au déroulement de l'évaluation, ce qui lui procure une bonne fiabilité intraet interjuges. Un autre atout de cette échelle se situe dans l'intégration des observations et informations provenant de la famille, des proches et de l'équipe soignante, que ce soit concernant les habitudes du patient précédant l'accident ou les comportements observés au cours de l'hospitalisation. Ces informations sont d'une grande importance pour l'évaluation et peuvent influer sur le choix des stimulations administrées au patient lors de la phase de traitement.

Par ailleurs, en tant qu'échelle comportementale, la SMART fait preuve de certaines limites. En effet, son utilisation clinique est difficile, non seulement par le temps d'administration de l'échelle qui s'élève à environ 45 minutes, mais aussi car le nombre d'évaluations et le temps nécessaire à l'obtention d'un diagnostic sont longs. Elle n'est donc, par exemple, pas adaptée au contexte d'évaluation en unités de soins intensifs. De plus, les critères diagnostiques définis selon les cinq niveaux sont vastes et ne permettent pas un diagnostic précis. De futures études comparatives sont nécessaires afin d'étudier sa validité concourante avec des échelles standardisées utilisées en clinique telles que la FOUR, la WHIM et la GLS. Il semble particulièrement intéressant de la confronter à la CRS-R qui, selon nous, est actuellement l'échelle de référence. En effet, certaines différences dans le choix des items dans ces échelles impliquent une vérification de leur utilité dans l'évaluation diagnostique. De plus, une évaluation de l'effet de l'échelle en tant que traitement devra aussi être faite afin de connaître son utilité en réhabilitation.

\section{Conflit d'intérêt}

\section{Aucun.}

\section{Remerciements}

Ce travail a bénéficié du soutien du Fonds de la recherche scientifique (FRS), de la Commission européenne (DISCOS, Mindbridge, DECODER \& CATIA), de l'Action de recherche concertée (ARC-06/11-340), la McDonnell Foundation et de la Mind Science Foundation.
RÉ F É R E N C E S

Andrews K, Murphy L, Munday R, Littlewood C. Misdiagnosis of the vegetative state: retrospective study in a rehabilitation unit. Br Med J 1996;313:13-6.

Ansell BJ, Keenan JE. The Western Neuro Sensory Stimulation Profile: a tool for assessing slow-to-recover head-injured patients. Arch Phys Med Rehabil 1989;70:104-8.

Born JD. The Glasgow Liège Scale: prognostic value and evolution of motor responses and brain stem reflexes after head injury. Acta Neurochir (Wien) 1988;91:1-11.

Giacino J, Ashwal S, Childs N, Cranford R, Jennett B, Katz D, et al. The minimally conscious state: definition and diagnostic criteria. Neurology 2002;58:349-53.

Giacino J, Kalmar K, Whyte J. The JFK Coma Recovery ScaleRevised: measurement characteristics and diagnostic utility. Arch Phys Med Rehabil 2004;85:2020-9.

Gill-Thwaites H. The Sensory Modality Assessment Rehabilitation Technique - a tool for assessment and treatment of patients with severe brain injury in a vegetative state. Brain Inj 1997;11:723-34.

Gill-Thwaites H, Munday R. The Sensory Modality Assessment and Rehabilitation Technique (SMART): a comprehensive and integrated assessment and treatment protocole for the vegetative state and minimally responsive patient. Neuropsychol Rehabil 1999;9:305-20.

Gill-Thwaites H, Munday R. The sensory modality assessment and rehabilitation technique (SMART): a valid and reliable assessment for vegetative state and minimally conscious state patients. Brain Inj 2004;18:1255-69.

Laureys S, Owen A, Schiff D. Brain function in coma, vegetative state and related disorders. Lancet 2004;3:537-46.

Malkmus D, Booth B, Kodimer C. Rehabiliation of the head injured adult: comprehensive cognitive management: Professional Staff Association of Rancho Los Amigos Hospital. California: Downey; 1980.

Posner J, Saper C, Schiff N, Plum F. Plum and Posner's diagnosis of stupor and coma. New York: Oxford University Press; 2007.

Schnakers C, Giacino J, Kalmar K, Piret S, Lopez E, Boly M, et al. Does the FOUR score correctly diagnose the vegetative and minimally conscious states? Ann Neurol 2006;60:744-5.

Schnakers C, Majerus S, Giacino J, Vanhaudenhuyse A, Bruno M, Boly M, et al. A French validation study of the Coma Recovery Scale-Revised (CRS-R). Brain Inj 2008;22:786-92.

Schnakers C, Vanhaudenhuyse A, Giacino J, Ventura M, Boly M, Majerus S, et al. Diagnostic accuracy of the vegetative and minimally conscious state: Clinical consensus versus standardized neurobehavioral assessment. BMC Neurol 2009;9:35

Shiel A, Horn SA, Wilson BA, Watson MJ, Campbell MJ, McLellan DL. The Wessex Head Injury Matrix (WHIM) main scale: a preliminary report on a scale to assess and monitor patient recovery after severe head injury. Clin Rehabil 2000;14:408-16.

Teasdale G, Jennett B. Assessment of coma and impaired consciousness. A practical scale. Lancet 1974;2:81-4.

The Multi-Society Task Force on PVS. Medical aspects of the persistent vegetative state (1). N Engl J Med 1994;330:1499-508.

Vanhaudenhuyse A, Schnakers C, Brédart S, Laureys S. Assessment of visual pursuit in post-comatose sates: use a mirror. J Neurol Neurosurg Psychiatry 2008;79:223.

Wijdicks EF, Bamlet WR, Maramattom BV, Manno EM, McClelland RL. Validation of a new coma scale: the FOUR score. Ann Neurol 2005;58:585-93.

Wilson S, Gill-Thwaites H. Early indication of emergence from vegetative state derived from assessments with the SMART - a preliminary report. Brain Inj 2000;14:319-31. 